\title{
Laparoscopic pancreaticoduodenectomy at Dr George Mukhari Academic Hospital
}

\author{
NC Kalenga, ${ }^{*}$ O Mongale, ${ }^{1}$ T Mosasi, ${ }^{1}$ M Govender ${ }^{2}$ \\ ${ }^{1}$ Surgical Registrar, Department of Surgery, Sefako Makgatho Health Sciences University, Dr George Mukhari Academic \\ Hospital, Pretoria, South Africa \\ ${ }_{2}^{2}$ Surgical Consultant, Department of Surgery, Sefako Makgatho Health Sciences University, Dr George Mukhari Academic \\ Hospital, Pretoria, South Africa
}

Corresponding author: Nkomba Christophe Kalenga (christophe_kalenga@yahoo.fr)

\begin{abstract}
Summary: Laparoscopic pancreaticoduodenectomy (PD) represents the culmination of advanced laparoscopy. PD is a challenging procedure performed open with few centres worldwide offering the surgery using minimal access techniques (laparoscopic and robotic). ${ }^{1,2}$ We report our institution's first case of PD performed laparoscopically.
\end{abstract}

\section{Case report}

A 62-year-old female presented with obstructive jaundice (total bilirubin $584 \mu \mathrm{mol} / \mathrm{L}$ ). Endoscopic biliary drainage was performed revealing a distal common bile duct stricture A contrast enhanced $\mathrm{CT}$ confirmed a resectable pancreatic head mass. Additionally, it revealed aberrant arterial anatomy, namely an early bifurcation of the hepatic artery such that the right hepatic artery (RHA) descended a short distance inferiorly giving rise to the gastroduodenal artery (GDA) prior to ascending parallel to the common bile duct (CBD) (Figure 1). After a period of nutritional optimisation, a laparoscopic PD was performed. The total operating time was 11 hours with $50 \mathrm{ml}$ blood loss.

The patient developed a short period of delirium postoperatively due to hypernatremia but recovered and was discharged on postoperative day 28 . No pancreatic or biliary leak occurred.

\section{Technique}

The patient was placed in a supine position with the lower limbs apart. An arterial line, central venous line and nasogastric tube (NGT) were inserted. Five ports were used with a 30 degree camera. The camera position was changed as required to offer the best view for different steps of the operation. After excluding metastases, we dissected the hepatoduodenal ligament with a combination of hook diathermy and Harmonic $\mathrm{ACE}+7^{\circledR}$ (HA). Our previous experience had alerted us to the fact that one may not complete steps as often done in open surgery but that one may have to revisit an area to complete a step. This is usually due to minor bleeding obscuring vision; we place a swab instead of using an energy device to control the bleeding.
We then entered the lesser sac and ligated the right gastroepiploic and right gastric vessels. An Endo GIA ${ }^{\circledR}$ stapling device using a black $60 \mathrm{~mm}$ cartridge was used to transect the stomach after ensuring the position of the NGT. The duodenum was kocherized to expose the left renal vein (Figure 2a). The GDA was ligated with a Hem-o-lok ${ }^{\circledR}$ clip and cut. A retropancreatic tunnel (Figure $2 b$ ) was created and the pancreas parenchyma transected with the HA. The pancreatic duct was cut with scissors. The jejunum was staple transected $10 \mathrm{~cm}$ distal to the ligament of Treitz with an Endo GIA ${ }^{\circledR}$ stapling device and mobilised under the superior mesenteric artery (SMA) to the right side. The cystic duct and CBD were tied and cut with scissors. The pancreas was dissected off the SMA with the HA. The gallbladder was left attached for removal at the end as it was used for retraction of the liver.

The pancreaticojejunostomy was done as described by Poves et al. ${ }^{3}$ A 3/0 Prolene $\left(\right.$ Ethicon $^{\circledR}$ ) suture was used for the outer layers with a duct to mucosa anastomosis (Figure 3a) using interrupted 4/0 Maxon $\left({ }^{\circledR}\right)$ sutures. The hepaticojejunostomy was completed with 4/0 PDS (Ethicon ${ }^{\circledR}$ ) continuous suture (Figure $3 b$ ). The gastrojejunostomy was completed with $3 / 0$ Maxon $\left(\right.$ Covidien $\left.{ }^{\circledR}\right)$. The specimen was retrieved via a $5 \mathrm{~cm}$ midline incision. Two pencil drains were placed; one at the biliary and another at the pancreatic anastomosis.

\section{Discussion}

The laparoscopic approach to pancreatic surgery has gained popularity in the last decade, especially for left pancreatectomy which is considered feasible and safe without increasing cost. ${ }^{4}$ Laparoscopic PD evolved slower since the first report by Gagner and Pomp in 1994. ${ }^{5}$ The advantages of laparoscopic PD in shorter time to discharge and less blood 


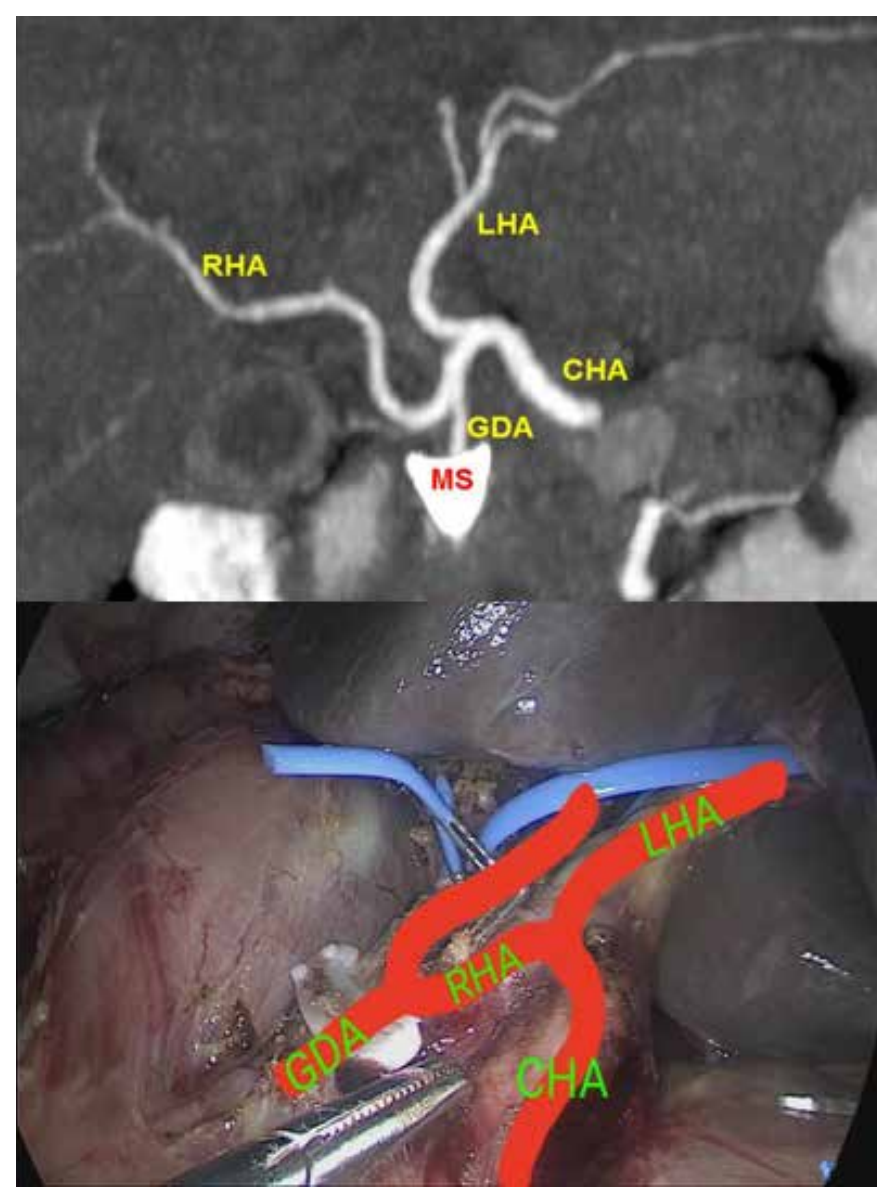

Figure 1: CT showing aberrant arterial anatomy and corresponding intraoperative findings. $M S=$ metal stent, $R H A=$ right hepatic artery, $L H A=$ left hepatic artery, $C H A=$ common hepatic artery, GDA = gastroduodenal artery

loss that have been reported in case series and meta-analyses have been confirmed in a recently published randomized trial. ${ }^{6}$ Retrospective comparative studies have shown similar oncological outcomes for open and laparoscopic PD in terms of survival and tumour-free margin status. Interestingly a higher lymph node yield is reported with the laparoscopic approach. ${ }^{4,5,6,7}$

This case highlights the importance of thorough preoperative evaluation of the patient, the anatomy and pathology as tactile sensation aiding in identifying especially vascular stricture in open surgery is absent in the total laparoscopic approach. At our institution it has taken ten attempted laparoscopic PDs before the procedure could be completed laparoscopically. We do agree that experience in performing the open procedure is essential. Previously we started the operation laparoscopically and proceeded to a point where the surgeon failed to progress or deemed further dissection unsafe. It was apparent that the lower blood loss and the smaller access used to complete these hybrid operations contributed to earlier recovery. Reasons for converting included the need to perform portal vein resection, bleeding and malrotation. We did not have the vast experience in performing less complicated laparoscopic pancreatic resections, such as left pancreatectomy as reported

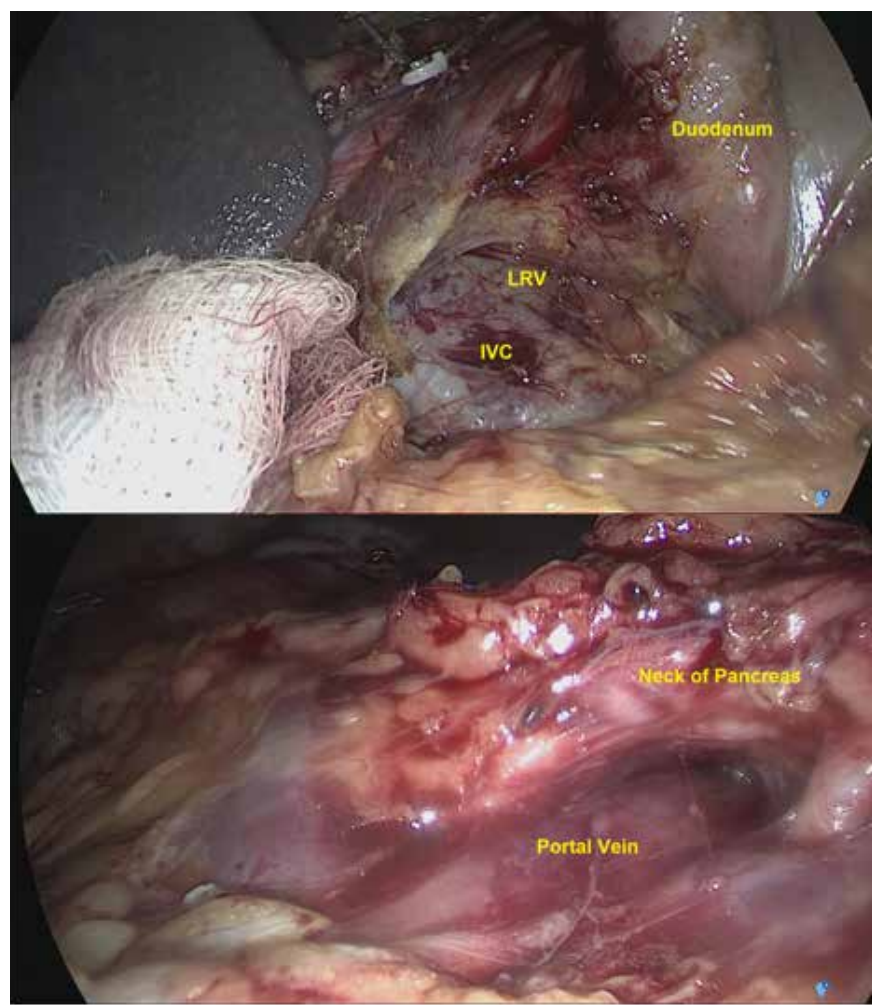

Figure 2a: Kocherization exposing inferior vena cava (IVC) and left renal vein ( $L R V)$

Figure 2b: Retropancreatic tunnel. Traction is provided by a suture on the inferior border (this also controls the inferior transverse pancreatic artery)

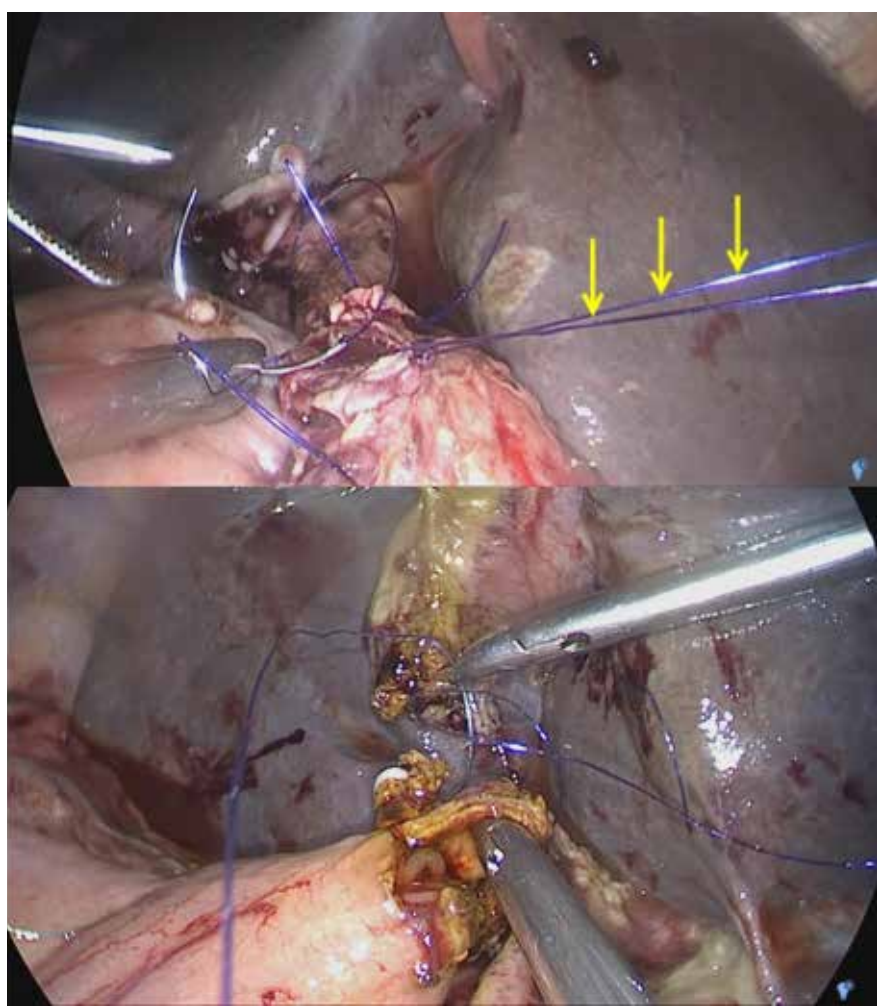

Figure 3a: Duct-to-mucosa anastomosis. The anterior suture (arrows) is placed first to provide traction, followed by the posterior suture (needle)

Figure 3b: Hepaticojejenostomy 
by other authors. ${ }^{7}$ This cumulative experience paved the road to the successful completion of our first laparoscopic PD at Dr George Mukhari Academic Hospital.

\section{Conclusion}

Laparoscopic PD is technically feasible despite the difficult learning curve. This approach is an extension of tools available to the hepatopancreaticobiliary surgeon. Knowledge of minimal access techniques alone will not compensate for lack of experience in pancreatic surgery and perioperative care. Worldwide trends and the advantages of the laparoscopic approach necessitate encouragement and further development of the technique in South Africa.

\section{REFERENCES}

1. Pędziwiatr M, Małczak $\mathrm{P}$, et al. Minimally invasive versus open pancreatoduodenectomy - systematic review and meta-analysis. Langenbecks Arch Surg. Aug 2017; 402(5):841-51.

2. Camilo CG, Dinkelspiel HE, et al. Minimall-Invasive vs Open Pancreaticoduodenectomy: Systemic Review and MetaAnalysis. J Am Coll Surg. Jul 2014; 218(1):129-39.

3. Poves I, Morató $\mathrm{O}$, et al. Laparoscopic-adapted Blumgart pancreaticojejunostomy in laparoscopic pancreaticoduodenectomy. Surg Endosc. Jul 2017;31(7):283745.

4. Hilal MA, Hamdan A, et al. Laparoscopic versus open distal pancreatectomy: a clinical and cost-effectiveness study. Surg Endosc. Jan 2012;26(6):1670-4.

5. Gagner M, Pomp A. Laparoscopic pylorus-preserving pancreaticoduodenectomy. Surg Endosc. May 1994;8(5):40810.

6. Palanivelu C, Senthilnathan P, et al. Randomized clinical trial of laparoscopic versus open pancreatoduodenectomy for periampullary tumours. BJS. Sep 2017;104(11):1443-50.

7. Palanivelu C, Jani K, et al. Laparoscopic pancreaticoduodenectomy: Technique and outcomes. J Am Coll Surg. Aug 2007;205(2):222-30. 